\title{
Necesidades de formación del profesorado de Lengua y Literatura para el desarrollo de la argumentación informal en el comentario de texto
}

\section{Training needs of Language and Literature teachers for the development of informal argumentation when doing text commentaries}

\author{
María Isabel De Vicente-Yagüe Jara ${ }^{1}$ \\ isabelvyague@um.es \\ María Teresa Valverde González \\ mariateresa.valverde@um.es \\ María González García \\ mgonzalezgarcia@um.es \\ Universidad de Murcia, España
}

\section{Resumen:}

Este estudio se enmarca en un proyecto de I+D+i sobre la formación del profesorado de Español como lengua materna y extranjera en la didáctica de la argumentación informal implicada en el comentario de texto, el cual ha sido patrocinado por el Ministerio de Economía, Industria y Competitividad del Gobierno de España. El objetivo general de la presente investigación consiste en profundizar en las demandas de formación y de materiales didácticos por parte de este profesorado para el desarrollo de la argumentación informal en el comentario de texto.

La investigación se centra en una metodología cualitativa de diseño interpretativo-fenomenológico para explorar los significados

\begin{abstract}
:
This study is part of an $\mathrm{R}+\mathrm{D}+\mathrm{i}$ project on the training of teachers of Spanish as a first and foreign language in the didactics of informal argumentation involved in text commentary, which was sponsored by the Ministry of Economy, Industry and Competitiveness of the Government of Spain. The general aim of this research consists of delving into the demands of training and teaching materials for the development of informal argumentation when doing text commentary.

The research adopts a qualitative methodology with an interpretative-phenomenological design in order to explore the meanings that explain the aforementioned demands. 34 teachers were selected, and
\end{abstract}

1 Dirección para correspondencia (correspondence address):

María Isabel de Vicente-Yagüe Jara. Departamento de Didáctica de la Lengua y la Literatura. Facultad de Educación. Universidad de Murcia. Campus de Espinardo, s/n. 30100 Espinardo (España). 
Necesidades de formación del profesorado de Lengua y Literatura para el desarrollo de la argumentación informal en el comentario de texto

María Isabel De Vicente-Yagüe Jara, María Teresa Valverde González y María González GarCíA

que explican las demandas aludidas, por medio del instrumento de la entrevista realizada a 34 docentes, que han sido seleccionados como informantes clave en atención a su perfil y experiencia profesional. El proceso de análisis se ha llevado a cabo mediante la reducción de la información obtenida en un mapa de significados, a través de un sistema inductivo de categorización y codificación de los datos realizado por el programa de análisis cualitativo Atlas.ti 7. Finalmente, el diálogo propiciado a través de las entrevistas ha revelado argumentos de gran interés sobre las demandas de formación y de materiales didácticos que este profesorado formula con razones causales y con la voluntad de autentificar su desarrollo profesional competente en didáctica de la argumentación informal en el comentario de textos.

\section{Palabras clave:}

Argumentación; comentario de texto; formación del profesorado; Lengua y Literatura; español. later interviewed, as key informants according to their profile and professional experience. Data analysis was conducted by means of the reduction of the information obtained in a map of meanings, through an inductive system of data categorisation and codification carried out with the qualitative analysis program Atlas.ti 7.

Finally, the dialogue fostered through the interviews revealed arguments of great interest regarding the demands of training and teaching materials, which these teachers formulate with causal reasons and with the will to authenticate their competent professional development in the didactics of informal argumentation in text commentary.

\section{Key words:}

Argumentation; text commentary; teacher training; Language and Literature; Spanish.

\section{Résumé:}

Cette étude est encadrée par un projet de $\mathrm{I}+\mathrm{D}+\mathrm{i}$ quant à la formation du professorat d'espagnol comme langue maternelle et étrangère en didactique de l'argumentation non-formelle impliquée dans le commentaire de texte. Cette étude a été soutenue par le Ministère de l'économie, de l'industrie et de la compétitivité du gouvernement espagnol. L'objectif principal de cette recherche consiste à approfondir les demandes en formation et en matériels didactiques de la part des professeurs, afin de développer l'argumentation non-formelle dans le commentaire de texte.

Cette recherche se base sur une méthodologie qualitative de conception interprétativephénoménologique dans le but d'explorer les éléments qui expliquent les demandes mentionnées; et ce, grâce à un entretien réalisé auprès de 34 enseignants, sélectionnés comme informateurs clé selon leur profil et leur expérience professionnelle. Le processus d'analyse a été réalisé après réduction de l'information obtenue sur une carte de sens via un système inductif de catégorisation et de codification des données, établi par le programme d'analyse qualitatif Atlas.ti 7 .

Finalement, le dialogue encouragé lors des entretiens a révélé des arguments de grand intérêt pour les demandes en formation et matériels didactiques. Cet ensemble de professeurs expose les motivations et la volonté de rendre authentique leur développement professionnel compétent en didactique de l'argumentation non-formelle dans le commentaire de textes.

\section{Mots clés:}

Argumentation ; commentaire de texte ; formation des professeurs ; Langue et Littérature ; espagnol.

Fecha de recepción: 28-2-2018

Fecha de aceptación: 5-3-2018 


\section{Justificación teórica}

En este artículo se presentan los resultados de una investigación exploratoria cuyo principal propósito ha sido esclarecer las demandas de formación y de provisión de recursos didácticos para el profesorado en relación con el desarrollo de la argumentación de carácter informal en el comentario de textos que se trabaja curricularmente en la etapa de Educación Secundaria. Dicha investigación se inserta en el proyecto de I+D+i "La formación en la argumentación informal del comentario de textos: diseño y análisis de un modelo competencial para la enseñanza del Español como lengua materna y lengua extranjera", con referencia institucional EDU201456997-P por parte del Ministerio de Economía, Industria y Competitividad (MINECO), que lo ha financiado desde el Programa Estatal de Fomento de la Investigación Científica y Técnica y de Innovación (2013-2016).

La finalidad de este proyecto es conocer y tratar el problema de la importante laguna investigadora existente sobre el tema que constituye su centro de interés, pues tal vacío ha ocasionado falta de respaldos científicos para estipular con suficiencia argumentativa modelos sólidos en formación específica del profesorado sobre dicho tema y, consecuentemente, deficiente desarrollo de la capacidad discente en escritura académica libre. Ante tal situación, este estudio fundamenta su teoría en el esclarecimiento conceptual de dos manifestaciones correlativas en el plano didáctico de la competencia en comunicación lingüística: la argumentación informal y el comentario de textos; ya que la formación en argumentación informal es estrategia de desarrollo educativo de la capacidad cognitivo-comunicativa que vincula aprendizajes formales e informales desde la discursividad dialógica, crítica y creativa del comentario personal de textos en el marco de las competencias clave 1 y 2 de la OCDE (2005).

En este marco, el presente estudio muestra los resultados de la investigación exploratoria sobre la percepción que el profesorado de Educación Secundaria especialista en Lengua Castellana y Literatura posee acerca de sus necesidades formativas y didácticas en relación con las prácticas argumentativas que deberían producirse habitualmente en el espacio discursivo del comentario de texto que se suele trabajar en el aula durante esta etapa. Esta investigación es pionera en su ámbito de estudio específico, pues, aunque el estado de la cuestión actual en autoeficacia docente lo convierte en un tema de gran interés en el horizonte de las competencias profesionales en materia educativa —según ha mostrado el Informe TALIS 
Necesidades de formación del profesorado de Lengua y Literatura para el desarrollo de la argumentación informal en el comentario de texto

María Isabel De Vicente-Yagüe Jara, María Teresa Valverde González y María González GarCíA

2013 para el desarrollo de la capacidad crítica (MECD, 2014, p. 132)—, no existen todavía publicaciones científicas que le antecedan al respecto. Ello no merma su interés, antes bien lo acrecienta, pues una de las demandas formativas más necesarias para el profesorado es "fomentar la lectura y el comentario crítico de textos de los diversos dominios científicos y culturales" (González-López y Reche-Urbano, 2010, p. 386).

La necesidad de trabajar de un modo integrador y democrático el discurso argumentativo en la lectura y el comentario de textos va ligada al cambio de mentalidad sobre el autoconcepto de la propia profesión, que ha supuesto en el cambio de siglo el nuevo paradigma de las competencias que desecha patrones conductistas y se acoge a la reflexión y la interacción en situaciones estratégicas y contextualizadas. Ello cobró pujanza desde el Proyecto Tuning que dio sentido articulado a la competencia profesional desde los primeros años del proceso de Bolonia atendiendo a la tríada de competencias instrumentales, interpersonales y sistémicas (González y Wagenaar, 2006), también reconocidas en la estipulación de las competencias clave enunciadas por la Comisión Europea (2004) y en la estela institucional de los sucesivos informes Eurydice y proyectos de trabajo desarrollados al respecto para ensamblar la lógica de las competencias con el desarrollo curricular (Roegiers, 2007; De Ketele, 2008; Moya, 2008; Bolívar, 2010; Perrenoud, 2012). Todos coinciden en la estimación de que el aprendizaje basado en competencias se dirige tanto hacia el profesorado como hacia el alumnado, como un acto de metacognición para la mejora de la calidad de sus actuaciones hacia la desenvoltura vital y la transferencia de conocimiento asociando aprendizajes formales e informales.

No obstante, a pesar de los esfuerzos institucionales referidos y de la sana crisis de identidad que ello produce en el profesorado afincado en el rol de transmisor de conocimiento de su disciplina para estimular su reconversión pedagógica hacia el paradigma competencial de la interdisciplinariedad desde una ética democrática (Bolívar, 2007; Escudero, 2009), a lo que se añade el reto de las nuevas alfabetizaciones digitales que demanda la Sociedad del Conocimiento (Salinas, De Benito y Lizana, 2014; Colás, Pablos y Ballesta, 2018; Castañeda, Esteve y Adell, 2018), sigue existiendo en el profesorado de Educación Secundaria un estilo ejecutivo (proclive a dar instrucciones a los estudiantes) y jerárquico (gusto por la planificación sin dejar margen a la improvisación), cuya rigidez suele frenar la autonomía y la creatividad discente (Doménech, 2012). Es por ello que se necesita insistir en el cultivo, durante la forma- 
ción inicial y permanente del profesorado de esta etapa educativa, de la reflexión crítica sobre su propio ejercicio en el desarrollo de las competencias clave que dan sentido a las enseñanzas formales en aprendizajes informales (Marcelo y Vaillant, 2009; Serrano y Pontes, 2016).

La práctica argumentativa es recuperada en este estudio desde su vertiente informal procuradora de competencia comunicativa con proyección epistémica por medio de razonamientos basados en inferencias que, con intención persuasiva, aportan pruebas y valores como apoyo de una conclusión (Johnson, 1999; Weston, 2005; Caro, 2018). A diferencia de la abstracción deductiva que caracteriza los silogismos de la lógica formal (Peralta, 2016), la lógica informal afronta acciones argumentativas sucedidas en circunstancias cotidianas (Bermejo, 2017). El paso de la lógica formal a la informal puede resumirse en el paso del cálculo a la crítica (Plantin, 2005).

El giro epistemológico de la argumentación hacia la reivindicación de su práctica informal advino con las teorías dialécticas de Toulmin (1958) sobre el tema, que pronto obtuvieron amplia difusión en la comunidad científica y, sobre todo, en los partidarios de la Pragma-dialéctica (Eemeren, Grootendorst y Shoecks Henkermans, 2002; Eemeren, 2011; Rodríguez, 2004; Bermejo, 2011; López, 2012; Marraud, 2013; Leal, 2016). Su teoría exponía la argumentación como acción de generar, plantear, impugnar, criticar o refutar tesis. A la propuesta de Toulmin se suma en contrapunto la de Perelman y Olberchts-Tyteca (1958), quienes abogan por el poder persuasivo de la argumentación ante su auditorio y el empleo de técnicas argumentativas neorretóricas. Entre las citadas vertientes dialéctica y neorretórica han proliferado indagaciones sobre la construcción gramatical de los argumentos (Lo Cascio, 1998), su pragmática lingüística (Grize, 1982; Doury, 2016) y multimodalidad (Adam y Bonhomme, 2000; Gómez, 2017).

También se recupera en este estudio la práctica democrática del comentario de texto que supera los modelos academicistas centrados en el significado del texto y su intención autorial que dejaron huella en España durante el siglo XX (Correa y Lázaro, 1957; Domínguez, 1977; DíezBorque, 1977; García Posada, 1982; Ariza, Guerrero y Torres, 1981) y acoge un enfoque semiótico y sociocrítico que activa la interpretación y el sentido personalizados contemplando, desde una metodología dialógica atenta a la polifonía de perspectivas (Ducrot, 1988) y a la procesualidad discursiva (Dijk, 1997; Björk y Blomstrand, 2000), tanto el enunciado y su contexto autorial como la enunciación y su contexto 
Necesidades de formación del profesorado de Lengua y Literatura para el desarrollo de la argumentación informal en el comentario de texto

María Isabel De Vicente-Yagüe Jara, María Teresa Valverde González y María González GarCíA

de retroalimentación entre emisor y receptor que corresponde (Romera, 1977; Bordons, Castellà y Costa, 1998; Álvarez, 2001; Cassany, 2008; Tusón y Vera, 2009; Caro y González, 2012).

Este posicionamiento epistemológico sobre la argumentación informal y el comentario de textos desde una clave competencial sirve de base teórica para contrastar su perfil con el que emerge de la consulta razonada al profesorado de Educación Secundaria al respecto a través de entrevistas donde, como se verá a continuación, el diálogo ha propiciado argumentos de gran interés sobre las demandas de formación y de materiales didácticos que este profesorado formula con razones causales y con la voluntad de autentificar su desarrollo profesional competente en didáctica de la argumentación informal en el comentario de textos.

Atendiendo a lo anteriormente expuesto, el objetivo general que se persigue en el presente estudio va dirigido a profundizar en las demandas de formación y de materiales didácticos por parte del profesorado para el desarrollo de la argumentación informal en el comentario de texto. Este objetivo general se concreta en los siguientes objetivos específicos:

1. Explorar las demandas de formación profesional en didáctica de la argumentación, atendiendo a las siguientes facetas: modelos teóricos, construcción lógico-sintáctica de los argumentos, retórica y pragmática.

2. Explorar las demandas de formación profesional en didáctica de la argumentación en el comentario de texto, atendiendo a las siguientes facetas: comentario de textos multimodales y evaluación de la competencia argumentativa.

3. Explorar las demandas de formación profesional en el diseño de materiales y recursos en formato impreso y digital.

4. Conocer otras necesidades profesionales sobre metodología docente de argumentación informal en el comentario de texto.

\section{Método}

La investigación se centra en una metodología cualitativa de diseño interpretativo-fenomenológico para profundizar en los significados que explican las demandas de formación del profesorado. Se ha accedido a la información por medio de entrevistas con los docentes, con la intención de recoger las características claves de su perfil profesional (León y Montero, 2015). 
Necesidades de formación del profesorado de Lengua y Literatura para el desarrollo de la argumentación informal en el comentario de texto María Isabel De Vicente-Yagüe Jara, María Teresa Valverde González y María González

García

\section{Participantes}

Los participantes de la investigación son un conjunto de 34 docentes del ámbito de Lengua y Literatura, seleccionados como informantes clave en atención a su perfil docente y experiencia profesional. Sus características sociodemográficas y profesionales se describen en la Tabla 1 (se comprueba cómo en los casos de la formación y la docencia impartida, diferentes docentes se incluyen en varios de los supuestos ofrecidos, lo que conlleva que en la tabla se muestre concretamente en esas situaciones un número total superior al de participantes).

Tabla 1. Participantes de la investigación.

\begin{tabular}{lll}
\hline Edad & 25-30 años & 2 \\
& $31-40$ años & 7 \\
& $41-50$ años & 8 \\
& $51-60$ años & 11 \\
& $61-70$ años & 6 \\
\hline Formación & Diplomatura & 2 \\
& Licenciatura & 26 \\
& Máster & 7 \\
& Doctorado & 14 \\
\hline Experiencia docente & $2-10$ años & 8 \\
& $11-20$ años & 11 \\
& $21-30$ años & 6 \\
& $31-40$ años & 9 \\
\hline Docencia impartida & Bachillerato & 26 \\
& Máster en Formación del Profesorado & 12 \\
& Español como Lengua Extranjera & 2 \\
\hline Titularidad del centro & Público & 32 \\
& Concertado & 2 \\
& Privado & 0 \\
\hline Estado del centro & Laico & 32 \\
& Religioso & 2 \\
\hline Bilingüismo del centro & Bilingüe & 28 \\
& No bilingüe & 6 \\
\hline
\end{tabular}


Necesidades de formación del profesorado de Lengua y Literatura para el desarrollo de la argumentación informal en el comentario de texto

María Isabel De Vicente-Yagüe Jara, María Teresa Valverde González y María González GarCíA

\section{Instrumentos}

Fue diseñada una entrevista por el grupo de investigadores del proyecto, en función de los objetivos planteados. Su contenido fue validado mediante el juicio de expertos (concretados en tres especialistas en el área de Didáctica de la Lengua y la Literatura, y dos especialistas de Métodos de Investigación y Diagnóstico en Educación), lo que permitió discutir las valoraciones ofrecidas y matizar la adecuación conceptual y lingüística del instrumento.

La estructura de la entrevista se divide en cuatro secciones diferenciadas: a) presentación; b) datos de carácter sociodemográfico, académico y profesional; c) cuerpo de cuestiones (Tabla 2); d) observaciones.

Tabla 2. Cuerpo de la entrevista.

Importa conocer las demandas académicas que usted percibe acerca de la metodología adecuada para desarrollar la argumentación informal en el comentario de textos.

1. Le vamos a proponer una serie de demandas de formación y de materiales didácticos para el desarrollo de la argumentación informal en el comentario de texto. Por favor, indíquenos si usted percibe también esta necesidad formativa y el porqué.

a) Modelos teóricos de la didáctica de la argumentación.

b) Construcción lógico-sintáctica de los argumentos (deductivos, abductivos, causales, etc.).

c) Retórica persuasiva de la argumentación.

d) Pragmática contextualizada de la argumentación.

e) Comentario de textos multimodales y sus estrategias argumentativas.

f) Evaluación de la competencia argumentativa demostrada en el comentario de texto.

g) Diseño de materiales y recursos digitales. ¿Cuáles?

h) Diseño de materiales y recursos impresos. ¿Cuáles?

2. Sugiera y explique otras necesidades profesionales que estime necesarias sobre metodología docente para la argumentación informal en el comentario de texto. 


\section{Procedimiento y plan de análisis de datos}

Las respuestas de los informantes clave fueron transcritas en soporte textual, con el fin de crear un documento primario de cada participante que diera lugar a un conjunto de unidades hermenéuticas, susceptibles de estudio mediante el programa de análisis cualitativo de datos Atlas.ti 7. El proceso de análisis se ha llevado a cabo mediante la reducción de la información obtenida en un mapa de significados, a través de un sistema inductivo de categorización y codificación de los datos (Flick, 2007), con la intención de dar sentido a la realidad expuesta por el profesorado acerca de sus demandas de formación para el desarrollo de la argumentación informal en el comentario de texto.

\section{Análisis de los resultados}

A continuación, se presentan los resultados de la investigación en función de los objetivos específicos formulados.

\section{Objetivo específico 1}

Con el fin de conocer las demandas de formación y de materiales didácticos del profesorado para el desarrollo de la argumentación informal en el comentario de texto, se ofrecieron ciertas necesidades, según se observa en el guión de la entrevista, para que los informantes clave comentaran si también percibían esa demanda y el porqué.

En primer lugar, según muestra la Figura 1, la demanda de modelos teóricos de la didáctica de la argumentación responde a la escasa formación de los docentes y, en algún caso, a una necesidad personal de aprender a enseñar. Disponer de modelos teóricos apropiados facilitaría mucho el trabajo del comentario en el aula. Estos modelos teóricos son necesarios como referente, aunque adaptables en cada contexto. Además, se debería contar con ejemplos que permitieran servir de guía en el desarrollo de la argumentación en el comentario crítico. Por otra parte, un docente señala que no ha profundizado en sus lecturas en esta modalidad, por lo que considera valiosa cualquier aportación de este tipo, destacando los modelos que lleven a trabajar la argumentación en textos 
Necesidades de formación del profesorado de Lengua y Literatura para el desarrollo de la argumentación informal en el comentario de texto

María Isabel De Vicente-Yagüe Jara, María Teresa Valverde González y María González GarCíA

literarios y publicitarios. Sin embargo, otro de los informantes clave remarca que no necesita ningún modelo teórico.

En segundo lugar, con respecto a la construcción lógico-sintáctica de los argumentos (deductivos, abductivos, causales, etc.), se matiza que se trata de procesos ligados a la reflexión metalingüística y, por tanto, relacionados con los conocimientos teórico-lingüísticos que se aplican en la práctica. Ese material ayudaría a conocer los tipos de argumentos que pueden emplearse desde un enfoque constructivo del discurso, "en lugar de teoría, acción verbal”, según afirma un informante-clave. Igualmente, se indica que se trata de una demanda de interés, aunque con margen para las aportaciones personales.

En tercer lugar, la retórica persuasiva de la argumentación es considerada como una demanda de gran importancia sobre la que no existen en la actualidad demasiados materiales didácticos disponibles; por ello, se suele trabajar normalmente de manera intuitiva. Además, se subraya que las estrategias retóricas entrañan ciertas dificultades para el alumnado. De igual modo, es necesario incidir en el grado de objetividad de las razones utilizadas, así como en la profundidad de la propia reflexión. Por el contrario, un docente comenta que, a su juicio, este apartado tiene menos interés, ya que la argumentación puede ser persuasiva, pero también tiene cabida en el discurso probatorio o expresivo.

En cuarto lugar, la pragmática contextualizada de la argumentación es una parte injustamente olvidada de la Lingüística en los currículos y, sin embargo, necesaria para la comprensión de textos. Resulta significativo realizar prácticas de lo aprendido, promoviendo el desarrollo de la riqueza argumentativa en situaciones concretas y con textos de diversas procedencias, ya que el hecho de acudir a tal variedad alejaría a los alumnos de las mecánicas conductistas donde no existe verdadera argumentación. Por otra parte, se trata de una demanda que conlleva cierta dificultad para el alumnado precisamente por su carácter pragmático. 
Necesidades de formación del profesorado de Lengua y Literatura para el desarrollo de la argumentación informal en el comentario de texto María Isabel De Vicente-Yagüe Jara, María Teresa Valverde González y María González

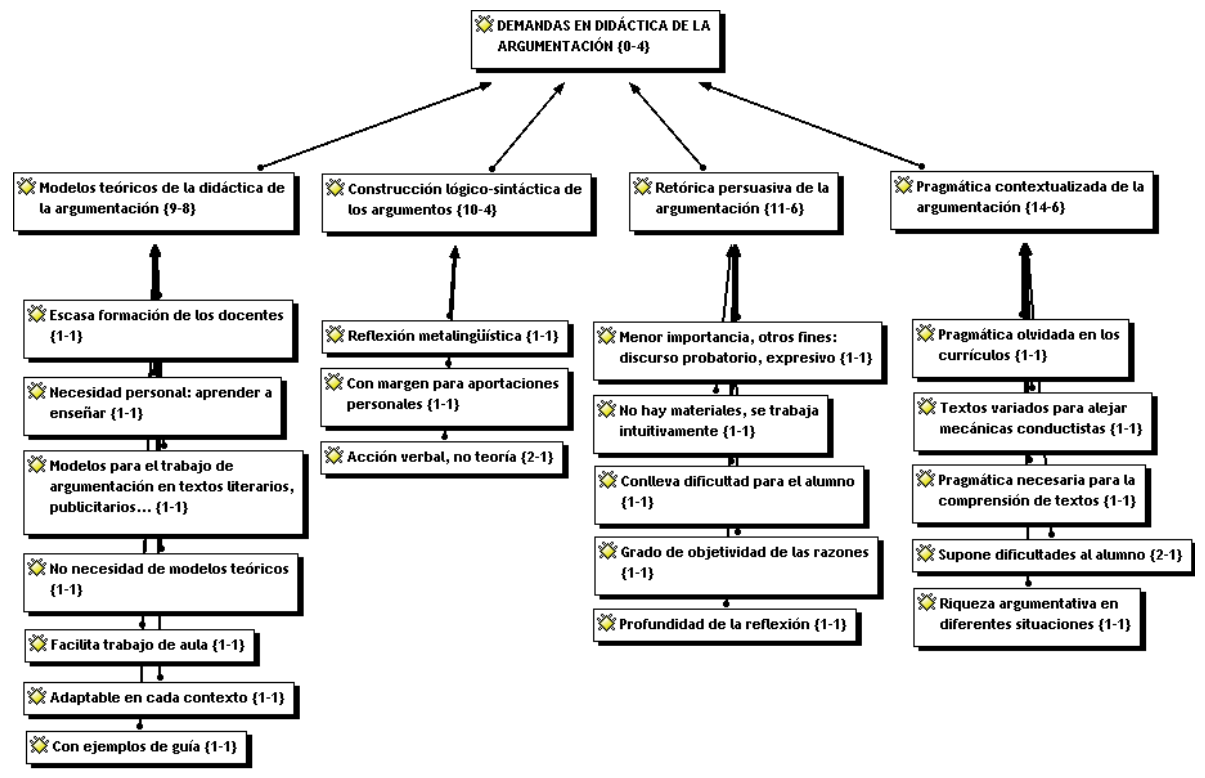

Figura 1. Red semántica de las demandas de formación profesional en didáctica de la argumentación.

\section{Objetivo específico 2}

Respecto de las demandas de formación profesional en didáctica de la argumentación en el comentario de texto (Figura 2), los docentes consideran fundamental la utilización de textos multimodales, así como trabajar la controversia, la argumentación y el desarrollo de su capacidad crítica en ellos y no únicamente en los textos periodísticos de opinión o los textos literarios, por lo que no extraña que esta propuesta de formación sea la más demandada por los entrevistados. Uno de los docentes puntualiza que aunque las estrategias se muestren de manera diferente en estos textos, deben responder también a la objetividad y no a la manipulación.

En cuanto a la demanda centrada en la evaluación de la competencia argumentativa demostrada en el comentario de texto, los profesores indican que sería necesario desarrollar recursos que facilitaran y concretaran este proceso de evaluación. Sin duda, el centro de atención de un modelo didáctico que gira en torno a la argumentación debe ser el proceso evaluador del mismo. Sin embargo, la evaluación objetiva 
Necesidades de formación del profesorado de Lengua y Literatura para el desarrollo de la argumentación informal en el comentario de texto

María Isabel De Vicente-Yagüe Jara, María Teresa Valverde González y María González GarCíA

de la competencia argumentativa es una tarea difícil y, por tanto, sería conveniente establecer una rúbrica precisa que ayude tanto al profesor como al alumno a medir los logros con descriptores, según se pide actualmente. Uno de los informantes-clave añade también que deberían ser evaluados de igual modo los efectos de la argumentación en los receptores. No obstante, un docente subraya que no cree necesario más indicaciones de evaluación, pues "ya tenemos bastante con una nueva ley que se ocupa más de la evaluación que de la enseñanza".

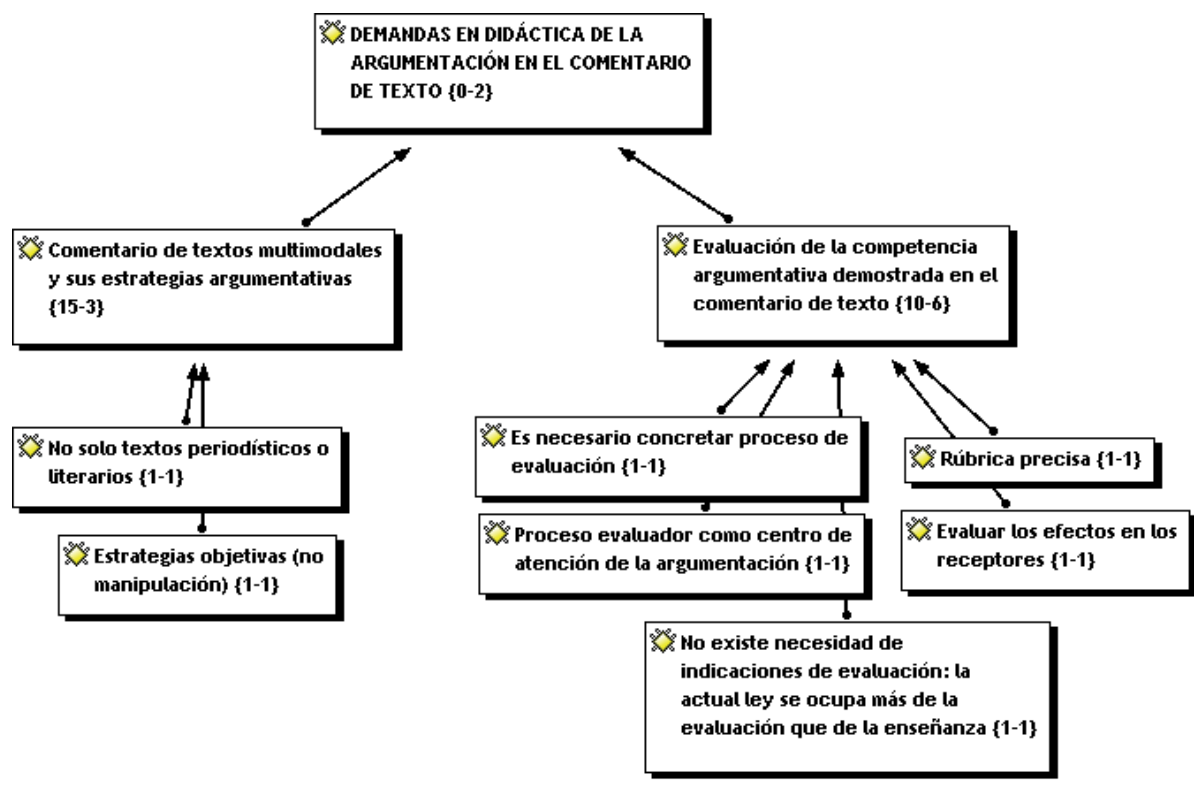

Figura 2. Red semántica de las demandas de formación profesional en didáctica de la argumentación en el comentario de texto.

\section{Objetivo específico 3}

Los docentes entrevistados demandan la elaboración de materiales y recursos didácticos tanto impresos como digitales (Figura 3). Debido a la escasez de materiales publicados, es necesario crear un banco de recursos para el aula. Se comenta la necesidad de una guía con gran variedad de ejercicios prácticos y de recursos para llevar directamente al aula, graduados por nivel de dificultad. Se puntualiza además la introducción de procesos y actividades tanto inductivos como deductivos. 
Por otra parte, los profesores solicitan la elaboración de manuales en los que se recojan textos en consonancia con los gustos de los alumnos y problemas de la sociedad. En este sentido, sería muy acertado recopilar una serie de vídeos cortos en los que los alumnos pudiesen observar los distintos tipos de argumentos: "pero no me refiero a vídeos de la BBC o de señores aburridos, sino fragmentos de series cercanas a ellos, películas, dibujos animados...", afirma un docente. Otro de los docentes destaca la utilidad del cine y los documentales como poderosos instrumentos para educar al alumnado en el desarrollo de la argumentación, y recuerda que no solo los textos escritos poseen esta función.

De igual modo, se demandan ejemplos de argumentaciones y diferentes comentarios de texto resueltos que sirvan de modelo a los alumnos previamente a la realización de su tarea didáctica. Es interesante también la creación de materiales interactivos para el progreso individual del alumno y el manejo de páginas web para tal fin.

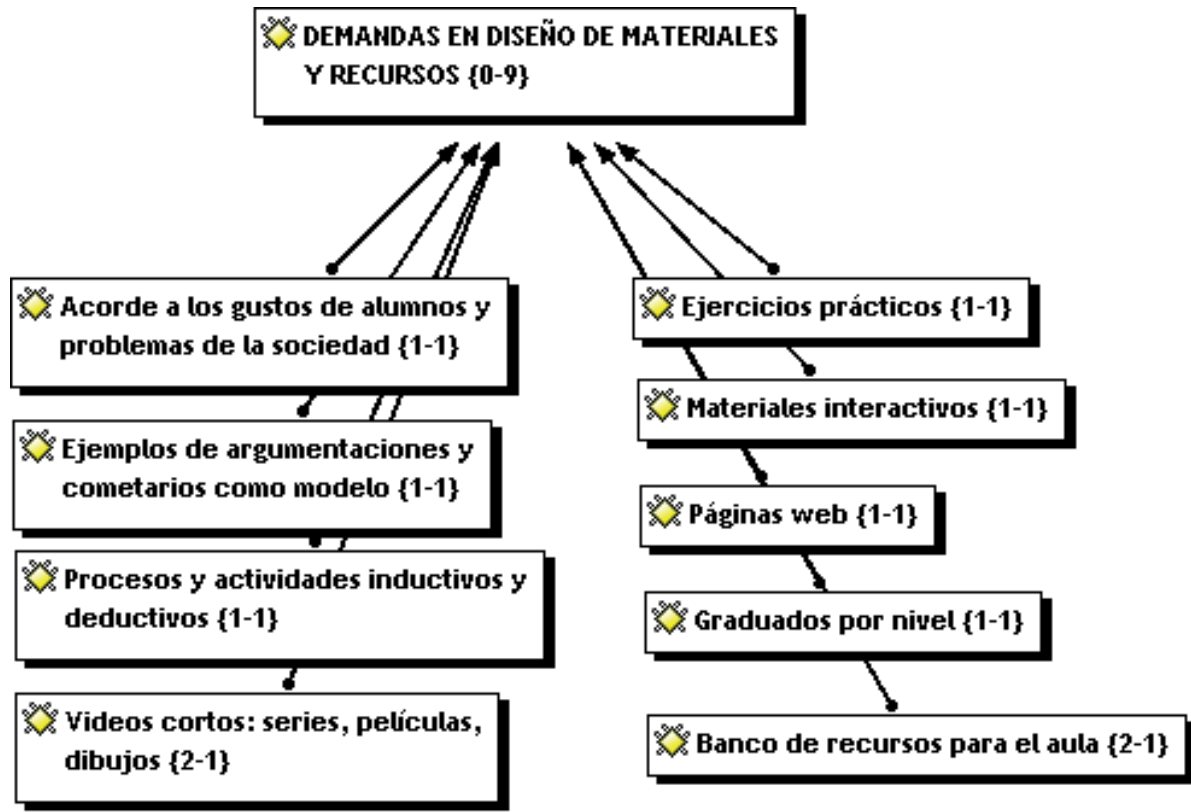

Figura 3. Red semántica de las demandas de formación profesional en el diseño de materiales y recursos. 
Necesidades de formación del profesorado de Lengua y Literatura para el desarrollo de la argumentación informal en el comentario de texto

María Isabel De Vicente-Yagüe Jara, María Teresa Valverde González y María González GarCíA

\section{Objetivo específico 4}

Finalmente, se pidió al profesorado que explicara otras necesidades profesionales, diferentes a las ya proporcionadas, que estimara necesarias sobre metodología docente de la argumentación informal en el comentario de texto, así como que aportara las sugerencias que considerara convenientes sobre el tema de estudio (Figura 4).

Se comenta la importancia de desarrollar un modelo dialógico de competencia comunicativa en el que la escritura, la lectura y la oralidad funcionaran correlativamente. Además, uno de los entrevistados demanda formación en la metodología del debate, pues entiende el debate como cauce de entrada a la mejora de la expresión escrita (así como al trabajo en equipo, liderazgo, investigación, entre otras ventajas educativas) y reconoce que no es una tarea sencilla de organizar. En esta línea, también se solicita el desarrollo de tareas cooperativas y del trabajo colectivo para procurar la construcción de archilecturas, la implicación, el debate, las valoraciones colectivas, el consenso en formulaciones finales y la construcción conjunta de argumentos.

Se cree conveniente la elaboración de propuestas de argumentación más concretas y explícitas en los libros de texto acordes a los diferentes niveles educativos; propuestas dirigidas incluso a la etapa de la Educación Secundaria Obligatoria. De todas formas, dado que la argumentación informal debe empezar a trabajarse desde Educación Primaria, de manera consecuente se plantea la necesaria formación del profesorado de esta etapa. Por otra parte, en cualquier actividad oral o escrita es conveniente tener en cuenta las cuestiones planteadas ya por Quintiliano y darles respuesta: qué, quién, cuándo, cómo, por qué y para qué; aunque pueda resultar obvio, no siempre es tarea fácil contestar estas preguntas.

De igual forma, se señala la utilidad de desarrollar la argumentación informal en el comentario de texto a través de metodologías fundamentadas en el aprendizaje basado en problemas y en el error como estrategia de aprendizaje. Por otro lado, con respecto a los procesos de evaluación, se cree conveniente desarrollar un modelo en el que la plantilla no rescate únicamente conductas sino valores logrados. Además, se sugiere la realización de pruebas individuales por medio de test, con el fin de poner en relación los conocimientos del alumnado con las posibles respuestas.

De modo general, se subraya la importancia de atender a la forma 
Necesidades de formación del profesorado de Lengua y Literatura para el desarrollo de la argumentación informal en el comentario de texto

María Isabel De Vicente-Yagüe Jara, María Teresa Valverde González y María González

GARCÍA

reflexiva de la argumentación, con el fin de potenciar que el alumno construya su propia opinión y no se apoye únicamente en los autores analizados tomados como cita de autoridad. Enseñar a pensar a los alumnos libremente de forma habitual es una tarea fundamental $y$, para ello, no es necesario plantear aspectos filosóficos de gran profundidad, sino únicamente cuestiones sencillas y corrientes: "los alumnos crecerían en libertad a la par que en sabiduría", según sostiene uno de los informantes-clave.

Para finalizar, otros docentes comentan, en relación con la excesiva carga de trabajo sobrellevada, la necesidad de encontrar métodos de corrección que no impliquen directamente al profesor, para poder de este modo desarrollar más frecuentemente tareas de escritura en el aula y reducir su labor de revisión. En los últimos años se ha elevado la ratio del alumnado en el aula, así como el horario lectivo del profesorado, lo que exige al docente con respecto a la tarea didáctica del comentario un excesivo tiempo de dedicación tanto en la labor de corrección como en la de guiar individualmente al alumno. Este hecho ha repercutido en que esta práctica didáctica no se realice de forma habitual y quede relegada en la mayoría de los casos al último curso de Bachillerato.

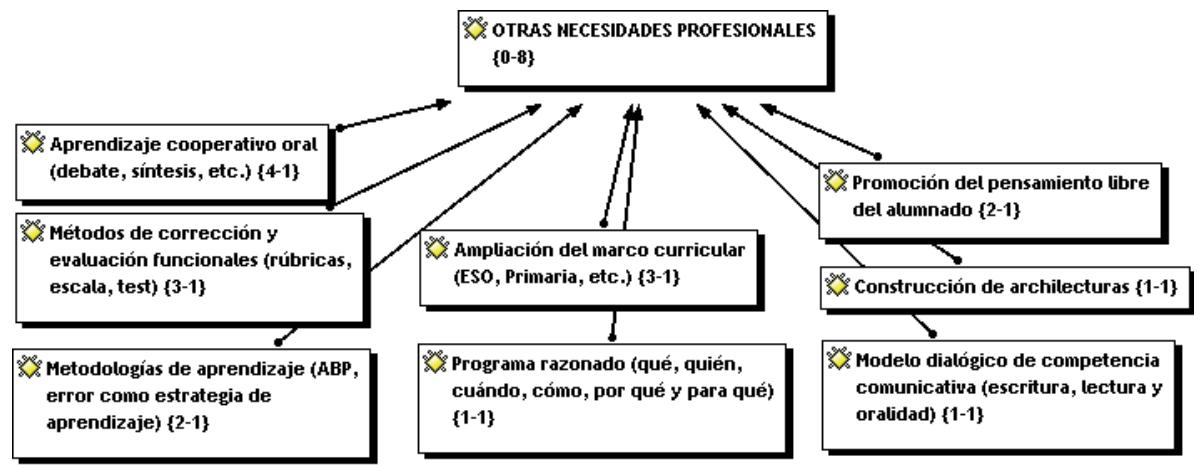

Figura 4. Red semántica de otras necesidades profesionales sobre metodología docente de la argumentación informal en el comentario de texto.

\section{Conclusiones}

Tras el análisis de los resultados, se comprueba que el diálogo a través de las entrevistas ha permitido la obtención de evidencias reales acerca 
Necesidades de formación del profesorado de Lengua y Literatura para el desarrollo de la argumentación informal en el comentario de texto

María Isabel De Vicente-Yagüe Jara, María Teresa Valverde González y María González GarCíA

de las demandas profesionales de formación y de materiales didácticos sobre argumentación informal en el comentario de textos, por medio de razones motivadas que persiguen el desarrollo profesional del docente.

En primer lugar, se descubre la creciente demanda de modelos teóricos sobre la didáctica de la argumentación informal por parte del profesorado. Ello responde a la marcada necesidad de guía razonada sobre el tema que tiene el docente de hoy, pues, como se aprecia en el marco teórico de este estudio, apenas existen modelos al respecto en el panorama editorial español que vayan dirigidos a las expectativas de enseñanza y aprendizaje de dicha modalidad discursiva en el área de Lengua castellana y Literatura. Obviamente, la satisfacción de esta necesidad contribuiría a mejorar la competencia crítica del alumnado a través de prácticas orientadas desde el mundo escolar. De igual modo, se destaca la reflexión metalingüística comentada en relación a la construcción lógico-sintáctica de los argumentos (Lo Cascio, 1998), el trabajo intuitivo en el aula de la retórica persuasiva de la argumentación (Perelman y Olberchts-Tyteca, 1958) y la necesidad de rescatar del olvido en los currículos oficiales a la pragmática contextualizada de la argumentación (Adam y Bonhomme, 2000), pues estas tres dimensiones (gramatical, retórica y pragmática) están íntimamente relacionadas en la construcción discursiva de los comentarios de texto en los que se desenvuelve la argumentación del comentarista. Si bien los avances curriculares de la última década sobre formación docente en la competencia en comunicación lingüística han hecho evolucionar al profesorado español hacia el trabajo educativo en la escala pragmalingüística del texto -y no en la escala tradicional de la frase- a la hora de establecer análisis y producción de discursos (MECD, 2009), no obstante, sigue siendo necesario formarlo en una visión clara de los procesos de planificación, textualización y revisión de tal ejercicio didáctico en modalidades discursivas concretas. Dicha situación evidencia la necesidad de generar y construir nuevos materiales y guías didácticas que apoyen buenas prácticas docentes de la argumentación alojada en el comentario de textos, además de los existentes (Caro y González, 2012), a fin de actualizar y enriquecer la formación profesional en este asunto con orientaciones didácticas precisas y certeras, un propósito al que, consecuentemente con estos resultados, perseguimos dar cumplimiento en el proyecto de $\mathrm{I}+\mathrm{D}+\mathrm{i}$ referido al inicio.

En segundo lugar, con respecto al desarrollo de la argumentación 
informal dirigida a la realización de tareas de comentario crítico personal, sobresale el empleo de textos multimodales para el trabajo de la capacidad crítica y pensamiento libre del alumnado como necesidad de formación más demandada por los docentes. Esta tendencia es coherente con la evolución innovadora marcada por los sucesivos informes PISA, que desde hace dos décadas abogan por el cultivo de la interpretación de textos continuos y discontinuos y de modalidades múltiples en formatos impreso y digital (OCDE, 2016). Por otro lado, se subraya el proceso evaluador de la competencia argumentativa demostrada en el comentario de texto como un centro de interés y, al mismo tiempo, se indica el empleo de rúbricas precisas que aseguren la objetividad en la evaluación. Este es otro de los retos más importantes esclarecidos en esta investigación, pues, como también queda indicado en la introducción teórica de este estudio, todavía queda mucho por investigar en didáctica del comentario argumentativo de textos desde la dimensión sistémica de su evaluación competente.

En tercer lugar, en consonancia con las demandas formuladas por los docentes comentadas anteriormente, las necesidades con respecto al diseño y la elaboración de materiales y recursos didácticos tanto impresos como digitales es manifiesta. Resulta destacable la mención a la necesidad de textos que se encuentren conectados con las preocupaciones vitales del alumnado, asunto imprescindible en una educación por competencias en la que se pretenden integrar los aprendizajes en un futuro contexto real de aplicación y entrenamiento del pensamiento crítico de los estudiantes. La interactividad digital, acorde con las existentes inquietudes digitales del alumnado, no deja tampoco indiferentes a los docentes encuestados. El profesorado demanda actualmente encontrar un terreno cómodo de acción en una materia como la del desarrollo de la argumentación informal en el comentario de texto, por lo que resulta coherente la necesidad explicada que se dirige a la creación de un banco de recursos para el aula, en la que se encuentren ejemplos de comentarios resueltos, entre otros materiales.

Por último, en alusión a las necesidades profesionales sugeridas por los docentes, se comprueba que no están relacionadas propiamente con el desarrollo de la argumentación como discurso lingüístico y lógico, sino con la estructura pedagógica idónea para permitir al docente llevar a cabo una práctica significativa y más adecuada. Cada alumno es particular y su nivel de desarrollo competencial hasta este momento es 
Necesidades de formación del profesorado de Lengua y Literatura para el desarrollo de la argumentación informal en el comentario de texto

María Isabel De Vicente-Yagüe Jara, María Teresa Valverde González y María González GarCíA

diverso al de sus compañeros, lo que ocasiona que el docente deba adecuar las estrategias didácticas y la metodología pedagógica, y que la tarea del comentario se reserve para ejecutarla en un tiempo ubicado fuera de los horarios contemplados por la Administración. En esta línea, se comenta la necesidad de bajar la ratio del alumnado, especialmente en la etapa de Bachillerato. Destaca en este último objetivo igualmente la idea comentada en relación a la construcción de archilecturas que el lector debe tejer, en consonancia con el concepto que Mendoza (2001) ya acuñaba de "intertexto lector" para referirse a una red de textos interconectada en la construcción del significado por parte de un lector competente.

Llegados a este punto, conocidas las demandas de los informantes clave entrevistados y siendo conscientes de la escasa literatura existente acerca del tema de estudio que nos ocupa, urge tomar medidas en el panorama de investigación del área de Didáctica de la Lengua y la Literatura y dar respuesta al profesorado preocupado en materia argumentativa y en cuestiones asociadas a la promoción del pensamiento crítico y libre del alumnado, asunto vital en el panorama actual de desarrollo de las competencias clave en discentes y docentes, desde un modelo dialógico de competencia comunicativa. En este sentido, el proyecto de I+D+i "La formación en la argumentación informal del comentario de textos: diseño y análisis de un modelo competencial para la enseñanza del Español como lengua materna y lengua extranjera", en el que, como se anunciaba inicialmente, se enmarcaba el presente estudio, pretende aportar vías y soluciones didácticas a las necesidades planteadas por los especialistas consultados en sus correspondientes objetivos de investigación.

\section{Referencias}

Adam, J. M y Bonhomme, M. (2000). La argumentación publicitaria. Retórica del elogio y de la persuasión. Madrid: Cátedra.

Almerich, G., Suárez-Rodríguez, J. M., Belloch, C. y Bo, R. M. (2011). Las necesidades formativas del profesorado en TIC: Perfiles formativos elementos de complejidad. RELIEVE. Revista Electrónica de Investigación Y Evaluación Educativa, 17(2), 1-28.

Álvarez, T. (2001). Textos expositivos-explicativos y argumentativos. Barcelona: Octaedro. Ariza, M., Garrido, J. y Torres, G. (1981). Comentario lingüístico literario de textos españoles. Madrid: Alambra Universidad. 
Necesidades de formación del profesorado de Lengua y Literatura para el desarrollo de la argumentación informal en el comentario de texto María Isabel De Vicente-Yagüe Jara, María Teresa Valverde González y María González

GARCÍA

Bermejo, L. (2011). Giving Reasons. A Linguistic-Pragmatic Approach to Argumentation Theory. London: Springer.

Bermejo, L. (2017). Lógica y teoría de la argumentación. Quadripartitaratio. Revista de retórica y argumentación, 2(4), 66-69.

Björk, L. y Blomstrand, I. (2000). La escritura en la enseñanza secundaria. Los procesos del pensar y del escribir. Barcelona: Graó.

Bolívar, A. (2007). La formación inicial del profesorado de secundaria y su identidad profesional. Estudios sobre Educación, 12, 13-30. Recuperado de https://dadun.unav. edu/bitstream/10171/8988/1/12\%20Estudios\%20Ea.pdf

Bolívar, A. (2010). Competencias básicas y currículo. Madrid: Editorial Síntesis.

Bordons, G., Castellà, J. M. y Costa, E. (1998). La lingüística textual aplicada al comentario de textos. Barcelona: Universidad de Barcelona.

Caro, M. T. (2018). La comunicación argumentativa en la Sociedad del Conocimiento, clave del liderazgo distribuido para un cambio educativo desde el desarrollo profesional. RED. Revista de Educación a Distancia, 56. doi: http://dx.doi.org/10.6018/ $\mathrm{red} / 56 / 8$

Caro, M. T. y González, M. (2012). Comentario de texto fácil para Bachillerato y acceso a la Universidad. Madrid: Espasa.

Cassany, D. (2008). Taller de textos. Leer, escribir y comentar en el aula. Barcelona: Paidós.

Castañeda, L., Esteve, F. y Adell, J. (2018). ¿Por qué es necesario repensar la competencia docente para el mundo digital?. RED. Revista de Educación a Distancia, 56(2). doi: http://dx.doi.org/10.6018/red/56/6

Colás, M. P., Pablos, J. de y Ballesta, J. (2010). Incidencia de las TI en la enseñanza en el sistema educativo español: una revisión de la investigación. RED. Revista de Educación a Distancia, 56(2). doi http://dx.doi.org/10.6018/red/56/2

Comisión Europea (2004). Competencias clave para un aprendizaje a lo largo de la vida. Un marco de referencia europeo. Bruselas: Dirección General de Educación y Cultura. Recuperado de http://www.educastur.princast.es/info/calidad/indicadores/doc/ comision_europea.pdf

Correa. E. y Lázaro, F. (1957). Cómo se comenta un texto en el Bachillerato. Grados elemental y superior curso preuniversitario. Salamanca: Anaya.

De Ketele, J. M. (2008). L'approche par compétences: au-delà du débat d’idées, un besoin et une nécessité d'agir. En M. Ettayebi, R. Operti y P. Jonnaert (Dir.). Logique de competences et développement curriculaire (pp. 61-78). Paris: L’Hatmattan.

Díez-Borque, J. M. (1977). Comentario de textos literarios (método y práctica). Madrid: Playor.

Dijk, T. van (1997). El Discurso como estructura y proceso. Barcelona: Gedisa.

Doménech, F. (2012). Análisis de los estilos de pensamiento que utilizan los profesores españoles en el aula. Revista de Educación, 358, 497-522. doi: 10-4438/1988-592XRE2010-358-088

Domínguez, J. (1977). Introducción al comentario de textos. Madrid: Servicio de Publicaciones del Ministerio de Educación y Ciencia.

Doury, M. (2016). Argumentation. Analyser textes et discours. Paris: Armand Colin.

Ducrot, O. (1988). Polifonía y argumentación. Colombia: Universidad del Valle. 
Necesidades de formación del profesorado de Lengua y Literatura para el desarrollo de la argumentación informal en el comentario de texto

María Isabel De Vicente-Yagüe Jara, María Teresa Valverde González y María González GarCíA

Eemeren, F. van (2012). Maniobras Estratégicas en el Discurso Argumentativo. Madrid: Plaza y Valdés-CSIC.

Eemeren, F. van, Grootendorst, R. y Shoecks Henkermans, F. (2002). Argumentation. Analysis, Evaluation, Presentation. London: IEA.

Escudero, J. M. (2009). La formación del profesorado de Educación Secundaria: contenidos de aprendizajes docentes. Revista de Educación, 350, 79-103. Recuperado de http://www.revistaeducacion.educacion.es/re350/re350_04.pdf

Flick, U. (2007). Introducción a la investigación cualitativa. Madrid: Morata.

García-Posada, M. (1982). El comentario de textos literarios. Madrid: Anaya.

Gómez, J. A. (2017). La argumentación jurídica: teoría y práctica. Madrid: Dykinson.

González, J. y Wagenaar, R. (2006). Una introducción a Tuning Educational Structures in Europe. La contribución de las universidades al proceso de Bolonia. Programa Socrates-Tempus. Recuperado de http://www.unideusto.org/tuningeu/images/stories/ documents/General_Brochure_Spanish_version.pdf

González-López, I. y Reche-Urbano, E. (2010). Las demandas formativas del alumnado de magisterio. Construcción de un plan de formación complementaria. Magis, 2(4), 383-400. Recuperado de http://hdl.handle.net/10396/8492

Grize, J. B. (1982). De la logique à l'argumentation. Ginebra: Droz.

Johnson, R. H. (1999). The relation between formal and informal logic. Argumentation, 13(3), 265-274.

Leal, F. (2016). "Reasonableness and Effectiveness in Argumentative Discourse" de Frans H. van Eemeren. Quadripartitaratio. Revista de retórica y argumentación, 1(1), 157 164.

Lo Cascio, V. (1998). Gramática de la argumentación. Madrid: Alianza editorial.

López, F. E. (2012). Las huellas pragmatistas en los usos de la argumentación. Cogency, $4(1), 25-51$.

Marcelo, C. y Vaillant, D. (2009). Desarrollo Profesional Docente. ¿Cómo se aprende a enseñar?. Madrid: Narcea.

Marraud, H. (2013). ¿Es lógic@? Análisis y evaluación de argumentos. Madrid: Cátedra.

MECD. (2009). Evaluación General de Diagnóstico 2009. Marco de la Evaluación. Madrid: Catálogo de publicaciones oficiales educación.es.

MECD. (2014). TALIS 2013. Estudio internacional de la enseñanza y el aprendizaje. Informe español. Madrid: MECD. Recuperado de https://goo.gl/ZJnnBm

Mendoza, A. (2001). El intertexto lector (El espacio de encuentro de las aportaciones del texto con las del lector). Cuenca: Ediciones de la Universidad de Castilla-La Mancha.

Moya, J. (2008). Las competencias básicas en el diseño y desarrollo del currículum. Revista Qurriculum, 21, 57-78.

Organización para la Cooperación y el Desarrollo Económicos. (2005). La definición y selección de competencias clave [Resumen ejecutivo] (Trad. Agencia de los Estados Unidos para el Desarrollo Internacional). Washington, DC: USAID.

Organización para la Cooperación y el Desarrollo Económicos. (2016). PISA 2015. Resultados clave. Recuperado de http://www.mecd.gob.es/dctm/inee/pisa-in-focus/pisa2015-results-in-focus-esp.pdf?documentld=0901e72b822a3ca6 
Necesidades de formación del profesorado de Lengua y Literatura para el desarrollo de la argumentación informal en el comentario de texto María Isabel De Vicente-Yagüe Jara, María Teresa Valverde González y María González

García

Peralta, V. M. (2016). ¿La lógica formal es útil para argumentar? La utilidad política. Quadripartitaratio. Revista de retórica y argumentación, 2(4), 24-29.

Perelman, C. y Olbrechts-Tyteca, L. (1989). Tratado de la argumentación. La nueva retórica. Madrid: Gredos.

Perrenoud, P. (2012). Cuando la escuela pretende preparar para la vida. ¿Desarrollar competencias o enseñar otros saberes?. Barcelona: Graó.

Plantin, C. (2005). L'argumentation. Paris: Puf.

Rodríguez, L. I. (2004). El modelo argumentativo de Toulmin en la escritura de artículos de investigación educativa. Revista Digital Universitaria, 5(1). Recuperado de http:// www.revista.unam.mx/vol.5/num1/art2/art2.htm

Roegiers, X. (2007). Pedagogía de la integración. Competencias e integración de los conocimientos en la enseñanza. San José: Coordinación Educativa y Cultural Centroamericana y $\mathrm{AECl}$.

Romera, J. (1977). El comentario de textos semiológico. Madrid: Sociedad General Española de Librería.

Salinas, J., De Benito, B. y Lizana, A. (2014). Competencias docentes para los nuevos escenarios de aprendizaje. Revista Interuniversitaria de Formación Del Profesorado, 79, 145-163.

Serrano, R. y Pontes, A. (2016). El desarrollo de la identidad profesional docente en la formación inicial del profesorado de Secundaria. Enseñanza y Teaching, 34, 1-2016, 35-55.

Toulmin, S. (1958). The Uses of Argument. Cambridge: Cambridge University Press.

Tusón, A. y Vera, M. (2009). El comentario de textos. Textos de Didáctica de la Lengua y la Literatura, 52, 5-10.

Weston, A. (2011). Las claves de la argumentación. Barcelona: Ariel. 
